ung thư gan (AFP, \% AFP-L3, PIVKA-II) có giá trị trung bình cao hơn ngưỡng. Các chỉ dấu sinh học ung thư gan không giúp loại trừ HCC, cân phối hợp thêm các phương pháp khác để chẩn đoán xác định.

\section{TÀI LIẸU THAM KHẢO}

1. Sung, H., Ferlay, J., Siegel, R. L., Laversanne, M., Soerjomataram, I., Jemal, A., \& Bray, F. (2021). Global Cancer Statistics 2020: GLOBOCAN Estimates of Incidence and Mortality Worldwide for 36 Cancers in 185 Countries. CA: a cancer journal for clinicians, 71(3), 209-249.

2. Yang, J. D., Hainaut, P., Gores, G. J., Amadou, A., Plymoth, A., \& Roberts, L. R. (2019). A global view of hepatocellular carcinoma: trends, risk, prevention and management. Nature reviews. Gastroenterology \& hepatology, 16(10), 589-604.

3. Galle, P. R., Foerster, F., Kudo, M., Chan, S. L., Llovet, J. M., Qin, S., ... Zhu, A. X. (2019). Biology and significance of alpha-fetoprotein in hepatocellular carcinoma. Liver international: official journal of the International Association for the Study of the Liver, 39(12), 2214-2229.

4. Pham Cẩm Phương, Mai Trọng Khoa và CS. (2019). Xét nghiệm AFP, AFP-L3 và PIVKA-II huyết thanh ở bệnh nhân ung thư biểu mô tế bào gan tại Bệnh viện Bạch Mai. Tạp chí Ung thư học Việt Nam. 2019, tr.301-306.
5. Lê Hữu Phước, Bành Vũ Điê̂n (2011). Đặc điểm lâm sàng và cận lâm sàng bệnh nhân ung thư tế bào gan (HCC) tai khoa nghiên cứu và điều tri viêm gan Bênh viện Chợ Rẫy. Tap chí Y học TP. Hồ Chí Minh. Tập 15(4)-2011: tr.570-572.

6. Phạm Thanh Bình, Hoàng Thị Minh và CS. (2018). Khảo sát đặc điểm lâm sàng, cận lâm sàng và nồng độ AFP, PIVKA-II ở bệnh nhân ung thư biểu mô tế bào gan. Tạp chí Y Dược thực hành 175. Tâp 15-2018: tr.60-67.

7. Nguyê̂n Trung Kiên, Nguyễn Thị Hồng Lê (2017). Khảo sát đăc điểm hình ảnh và giá trị của siêu âm, chup cắt lớp vi tính ung thư biểu mô tế bào gan tại Bệnh viện Quân Y 4. Tạp chí Y Dược học quân sự. Tập 1-2017: tr.105-111.

8. Kim, G. A., Kim, H. I., Chang, S., An, J., Lee, D., Lee, H. C., Han, S., \& Shim, J. H. (2019). A Prospective Evaluation of the Reliability and Utility of Quality of Life Measures in Patients With Hepatocellular Carcinoma. American journal of clinical oncology, 42(7), 555-563.

9. Chie, W. C., Yu, F., Li, M., Baccaglini, L., Blazeby, J. M., Hsiao, C. F., Chiu, H. C., Poon, R. T., Mikoshiba, N., Al-Kadhimi, G., Heaton, N., Calara, J., Collins, P., Caddick, K. Costantini, A., Vilgrain, V., \& Chiang, C. (2015). Quality of life changes in patients undergoing treatment for hepatocellular carcinoma. Quality of life research : an international journal of quality of life aspects of treatment, care and rehabilitation, 24(10), 2499-2506.

\title{
ĐÁNH GIÁ TÌNH TRANG DINH DƯÕ̃NG THEO THANG ĐO CỦA TRUNG TÂM DINH DƯỠNG TP.HCM ĐỐI VỚI THAI PHỤ ĐẾN SINH TẠI BỆNH VIỆN ĐA KHOA KHU VỰC PHÍA NAM BÌNH THUẬN
}

Đỗ Đình Trung ${ }^{1}$, Lê Văn Huỳnh ${ }^{1}$, Tô Mai Xuân Hồng ${ }^{2}$

TÓM TẮT

Đă̆t vấn đề: Chễ độ dinh dưỡng đầy đủ và đúng cách trong quá trình mang thai có vai trò quan trọng trong sự phát triển và tăng trưởng của thai nhi. Nghiên cứu tiến hành nhằm xác định tỳ lệ dinh dưỡng kém bằng thang đo dinh dưỡng của Trung tâm dinh dưỡng TPHCM. Phương pháp nghiên cứu: Thiết kế nghiên cứu mô tả cắt ngang từ $01 / 11 / 2020$ đến $31 / / 05 / 2021$. Sử dụng bảng thang đo dinh dưỡng cúa Trung tâm dinh dưỡng TPHCM trên 330 thai phụ đến sinh tại bệnh viện đa khoa khu vực Phía Nam Bình Thuận, với điểm cắt bằng 2,0 điểm để xác định thai phụ có nguy cơ dinh dưỡng. Kết quả: Thai phụ có nguy cơ dinh dưỡng chiếm 17,0\% (KTC 95\% 13,321,8). Kết cục xấu của mẹ: 2,1\% (KTC 95\%: 0,6 -

${ }^{1}$ Bệnh viện đa khoa khu vực Nam Bình Thuận

2 Trường đai hoc Y Dướ TPHCM

Chịu trách nhiệm chính: Tô Mai Xuân Hồng

Email: tomaixuanhong@ump.edu.vn

Ngày nhận bài: 11.5.2021

Ngày phản biên khoa học: 24.6.2021

Ngày duyệt bài: 12.5.2021
$3,9)$ bao gồm: tiền sản giật/sản giật $(1,5 \%)$, Băng huyết $(0,6 \%)$, nhau bong non $(0,6 \%)$, nhiễm trùng sau sinh $(0,3 \%)$. Kết cục xấu của con: $2,4 \%$ (KTC95\%: 0,9 - 4,2) bao gồm: can thiệp nhi $(0,6 \%)$, chuyển dương nhi $(2,1 \%)$, vàng da sau sinh $(0,9 \%)$, chuyển tuyến điều trị $(0,9 \%)$. Các thai phụ là ngưới dân tộc thiểu số có nguy cơ gặp bất thường về dinh dưỡng trong thai kỳ cao gấp 4,8 lần $(O R=4,8 ; K T C$ $95 \%: 1,01-22,5)$ so với thai phụ là người Kinh. BMI $<18,5$ trước khi sinh con làm tắng nguy cơ găp bất thường về dinh dưỡng gấp 64,1 lần (OR=64,1; KTC 95\%: 11,2-368,3). Có mối liên quan giữa kết cuc thai kỳ xâu và bất thường dinh dưỡng trong thai kỳ $(p<0,05)$. Kết luân: Sử dung thang đo dinh dương của Trung tâm dinh dưỡng TPHCM với điểm cắt 2,0 điểm giúp sàng lọc đối tượng thai phụ có nguy cơ rối loạn dinh dưỡng trong thai kỳ, và tiên đoán các ảnh hưởng đến sức khoẻ cho thai phu và bé sơ sinh.

Từ khóa: thang đo dinh dướng, dinh dưỡng trong thai kỳ, kết cục thai kỳ.

\section{SUMMARY \\ USING THE NUTRITIONAL SCALE OF HOCHIMINH NUTRITIONAL CENTER FOR}




\section{ASSESSING PREGNANT WOMEN WHO GIVING BIRTH AT A GENERAL HOSPITAL IN THE SOUTH OF BINH THUAN}

Background: An appropriate and healthy diet during pregnancy plays an important role to help fetus become growth and wellbeing. The study is aimed to determine the prevalence of poor nutrition by using the nutritional scale of Ho Chi Minh Nutritional Center. Methods: The cross-sectional descriptive study is carried out from November $1^{\text {st }} 2020$ to May $31^{\text {st }} 2021$. We applied the nutritional scale of Ho Chi Minh Nutritional Center to assess the nutrition status in 330 pregnant women before giving birth at the general hospital in the South of Binh Thuan. Pregnant women who are considered as risk of nutrition disorders at the cutoff at 2.0 according to $\mathrm{Ho}$ Chi Minh Nutritional Center. All pregnant women at risk are closely followed at delivery room and post-partum to evaluate further adverse maternal and neonatal outcomes. Results: The prevalence of pregnant women at risk for nutritional disorders is $17.0 \%$ (95\% CI 13.3-21.8). Maternal outcomes are 2.1\% (95\%CI:0.6-3.9) including preeclampsia/eclampsia (1.5\%), post-partum haemorrhage $(0.6 \%)$, placental abruption preterm birth $(0.6 \%)$, postpartum infection $(0.3 \%)$. Neonatal outcomes are found in 2.4\% (CI 95\%: $0.9-4.2$ ) including perinatal intervention $(0.6 \%)$, emergency of pediatric transfer $(2.1 \%)$, severe neonatal jaundice $(0.9 \%)$, referral for treatment $(0.9 \%)$. Pregnant women from ethnic minorities have 4.8 times higher risk for nutritional disorders pregnancy $(\mathrm{OR}=4.8 ; 95 \%$ CI: 1.01-22.5) than the ones who are in majority ethnics. Women with low body mass index (BMI $<18.5$ ) before getting pregnant are identifed 64.1 times higher risk for nutritional disorders than the ones with healthy body (OR=64.1; 95\% CI: $11.2-$ 368.3). There is a significant relation between the nutrition disorders during pregnancy and adverse outcomes at delivery and post-partum $(p<0.05)$. Conclusion: The nutritional scale of $\mathrm{Ho}$ Chi Minh Nutritional Center at the cutoff of 2.0 to screen pregnant women, who are at risk for nutritional disorders during pregnancy, and to predict the neonatal and maternal outcomes.

Keywords: Nutritional scale, nutritional disorders in pregnancy, adverse outcomes.

\section{I. ĐẶT VẤN ĐỀ}

Dinh dưỡng đầy đủ và đúng cách trong quá trình mang thai giữ vai trò quan trọng trong sự phát triển và tăng trưởng của thai nhi. Nhiều nghiên cứu đã chứng minh rằng thiếu hụt dinh dưỡng trong quá trình mang thai có thể làm tăng nguy cơ xuất hiện tai biến sản khoa và sinh ra trẻ non tháng cũ̃ng như trẻ nhẹ cân(1),(2). Khi thai phụ bị thiếu dinh dưỡng có thể hạn chế số lượng và chất lượng sữa mẹ, giảm nguồn cung cấp thức ăn lý tưởng nhất cho thai nhi, là nguyên nhân suy dinh dưỡng, tăng nguy cơ mắc bệnh và tử vong cho trẻ sơ sinh ${ }^{(1)}$.

Hiện nay các hướng dẫn về chế độ dinh dưỡng trong thai kỳ một cách chi tiết vẫn còn tản mạn, thiếu đồng bộ và nhất quán, chưa tập trung vào các thực hành cụ thể, từ đó, dẫn tới khó khăn cho nhấn viên y tể làm công tác tư vấn dinh dưỡng ${ }^{(1)}$. Nghiên cứu của Lê Thị Thanh Hoa và cộng sự năm 2014 xác định chỉ có 47,9\% phụ nữ mang thai chưa đạt mức tăng cân khuyến nghị của Viện Y học (3). Kết luận này đặt ra việc cần có nhiều nghiên cứu đánh giá tình trạng lại dinh dưỡng trong thai kỳ một cách cụ thể hơn cũng như xác định ảnh hưởng như thế nào đến kết cục thai kỳ.

Bệnh viện đa khoa khu vực Phía Nam Bình Thuận là nơi tập trung đông đồng bào dân tộc sinh sống. Trong đó, có từ $10-15 \%$ thai phụ là người dân tộc thuộc nhóm dinh dưỡng kém (nhe cân khi mang thai so với khuyến cáo của Bộ Y tế) (1). Bối cảnh này đặt ra một thách thức cho công tác chăm sóc sức khoẻ thai phụ nhằm nâng cao tình trạng dinh dưỡng của các thai phụ đến khám thai tai bệnh viện đa khoa khu vực Phía Nam tỉnh Bình Thuận. Với mong muốn cải thiện chương trình chăm sóc sức khoẻ thai phụ, dựa trên các hướng dẫn chi tiết về tình trạng dinh dưỡng trong thai kỳ, nghiên cứu được thực hiện nhằm đánh giá tình trạng dinh dưỡng ở các thai phụ đến sinh tại bệnh viện đa khoa khu vực phía Nam Bình Thuận dựa vào thang đo của Trung tâm dinh dưỡng TPHCM. chúng tôi tiến hành nghiên cứu nhằm "Đánh giá tình trạng dinh dưỡng theo thang đo của Trung tâm dinh dưỡng TPHCM đối với thai phụ đến sinh tại bênh viện đa khoa khu vực Phía Nam Bình Thuận". Muc Tiêu Nghiên Cứu: Xác định tỷ lệ dinh dưỡng kém bằng thang đo dinh dưỡng của Trung tâm dinh dưỡng TPHCM trên thai phụ đến sinh tại bệnh viện đa khoa khu vực Phía Nam Bình Thuận.

\section{II. ĐỐI TƯỢNG VÀ PHƯƠNG PHÁP NGHIÊN CứU}

- Thiết kế nghiên cứu: Nghiên cứu mô tả cắt ngang

- Đối tượng nghiên cứu

- Tiêu chuẩn lựa chọn:

+ Phụ nữ đủ 18 tuổi đến sinh tại khoa sản của bệnh viện

+ Có sổ khám thai hoặc được lưu trữ thông tin khám thai từ đầu thai kỳ tại bệnh viện đa khoa khu vực Phía Nam Bình Thuận

+ Hiểu và giao tiếp tốt được bằng tiếng Việt

+ Đồng ý tham gia nghiên cứu

- Tiêu chuẩn loại trừ:

+ Không đồng ý tham gia nghiên cứu

+ Không có sổ khám thai tai bệnh viện

+ Không hiểu và không giao tiếp được bằng 
tiếng Việt

Cỡ mẫu: Chọn mẫu toàn bộ

- Địa điểm và thời gian nghiên cứu

+ Địa điểm nghiên cứu: Khoa sản - Bệnh viện ĐKKV Phía Nam Bình Thuận

+ Thời gian nghiên cứu dự kiến: từ $01 /$ $11 / 2020$ - 31/ 05/2021

- Phương pháp chọn mẫu. Dựa trên phương pháp chọn mẫu ngẫu nhiên hệ thống: Hệ số ngẫu nhiên $\mathrm{k}=\mathrm{N}$ tổng số sản phụ đến sinh tại bệnh viện trong 6 tháng)/n (cõ mẫu), như vậyk $=1000 / 260=3,8$. Vì vậy, để đảm bảo thu thập đủ cõ mẫu trong nghiên cứu, chúng tôi chọn hệ số ngẫu nhiên $\mathrm{k}=3$.

- Phương pháp tiến hành. Tất cả các sản phụ đến sinh tại bệnh viện được lập danh sách khi vào sinh tại khoa sản theo phương pháp chọn mẫu ngẫu nhiên hệ thống. Sau đó, họ được phỏng vấn và tư vấn trước khi tiến hành thu thập số liệu. Chúng tôi ghi nhận các thông tin về dịch tể học, tình trạng mang thai và các số đo theo tiêu chí của thang đo dinh dưỡng do Trung tâm dinh dưỡng TPHCM ban hành. Các thai phụ có tình trang dinh dưỡng kém ở điểm cắt bằng 2,0 được theo dõi và ghi nhận kết cục thai kỳ của sản phụ và trẻ được sinh ra.

\section{-Biến số nghiên cứu}

+ Kết cục thai kỳ xấu của me, có bất kỳ 1 trong nhưng yếu tố sau: băng huyểt; sản giật; võ tử cung; nhiểm trùng sau sinh; uốn ván rốn sơ sinh; nhau bong non; chuyển tuyến/tử vong (4).

+ Kết cục thai kỳ xấu, có bất kỳ 1 trong nhưng yếu tố sau: can thiệp nhi; chuyển dưỡng nhi; trẻ vàng da; sử dụng kháng sinh điều trị; chết lưu; chuyển tuyến/tử vong.

+ Kết cuc tốt: không có bất kỳ những yếu tố kết cục xấu (4).

\section{- Tiêu chuẩn đánh giá}

Dựa trên tiêu chuẩn đánh giá của Trung tâm dinh dưỡng TPHCM phối hợp cùng UNICEF xây dựng tại Việt Nam (5):

\begin{tabular}{|c|c|c|}
\hline $\begin{array}{l}\text { BMI trước } \\
\text { mang thai }\end{array}$ & $\begin{array}{l}18,5-24,9 \\
\quad \geq 25 \\
\quad<18\end{array}$ & \begin{tabular}{|l} 
- 0 Điếm \\
- 1 điểm \\
- 1 điểm
\end{tabular} \\
\hline $\begin{array}{c}\text { Chu vi } \\
\text { vòng cánh } \\
\text { tay }\end{array}$ & $\begin{array}{l}\geq 23 \\
<23\end{array}$ & - 0 điểm \\
\hline $\begin{array}{l}\text { Tốc độ } \\
\text { tăng cẩn }\end{array}$ & $\begin{array}{l}\text { Tăng cân theo khuyến } \\
\text { nghị } \\
\text { Tăng cân, trên hoặc } \\
\text { dưới khuyến nghị }\end{array}$ & $\begin{array}{ll}\text { - } & 0 \text { Điểm } \\
\text { - } & 1 \text { điểm }\end{array}$ \\
\hline $\begin{array}{l}\text { Bềnh lý } \\
\text { kèm theo } \\
\text { liên quan }\end{array}$ & $\begin{array}{c}\text { Không } \\
\text { THA, ĐTĐ thai kỳ, } \\
\text { ngén nặng, thiếu máu }\end{array}$ & $\begin{array}{ll}\text { - } & 0 \text { Điểm } \\
\text { - } & 1 \text { điểm }\end{array}$ \\
\hline
\end{tabular}

\begin{tabular}{|c|c|c|}
\hline $\begin{array}{c}\text { đến dinh } \\
\text { dưỡng }\end{array}$ & $\begin{array}{c}\text { dinh dưỡng, bệnh lý } \\
\text { đường tiêu hóa, }, . .\end{array}$ & \\
\hline Kết luận & $<2$ điểm & $\begin{array}{c}\text { Bình } \\
\text { thường } \\
\text { Cón nguy } \\
\text { cơ rối loạn } \\
\text { dinh dưỡng }\end{array}$ \\
\hline
\end{tabular}

III. KẾT QUẢ NGHIÊN CỨU

3.1. Đặc điểm dinh dưỡng trong lân mang thai này

Bảng 1: Đặc điểm dinh dưỡng trong lân mang thai này



Nhận xét: Trong 330 thai phụ được khảo sát có thể tạng bình thường với chì số khối cơ thể (BMI) trung bình là 20,45 $\pm 1,7$ với BMI cao nhất là 33,75 và $B M I$ thấp nhất là 12,33 . $57,6 \%$ thai phụ tăng cân đúng khuyến cáo (trung bình $12 \mathrm{~kg}$ trong suốt thai kỳ). Chu vi vòng cách tay $<23 \mathrm{~cm}$ chỉ chiếm $1,5 \%$. Bệnh lý kèm phổ biến nhất là thiếu máu $7,6 \%$.

3.2. Tỷ lệ thai phụ có nguy cơ dinh dưỡng

Bảng 2: Dinh dướng của thai phù trong thai kyे

\begin{tabular}{|c|c|c|c|}
\hline Đặc điểm & $\begin{array}{c}\text { Tân suất } \\
(\mathbf{N = 3 3 0 )}\end{array}$ & $\begin{array}{c}\text { Tỷ lệ̂ } \\
(\mathbf{\%})\end{array}$ & $\begin{array}{c}\text { KTC95 } \\
\text { \% }\end{array}$ \\
\hline $\begin{array}{c}\text { Nguy cơ dinh } \\
\text { dương }\end{array}$ & 56 & 17,0 & $\begin{array}{c}13,3- \\
21,8\end{array}$ \\
\hline $\begin{array}{c}\text { Không nguy cơ } \\
\text { dinh dưỡng }\end{array}$ & 274 & 83,0 & $\begin{array}{c}78,2- \\
86,7\end{array}$ \\
\hline
\end{tabular}

Tỷ lệ thai phụ có nguy cơ dinh dưỡng được xác định bằng thang đo dinh dưỡng của Trung 
tâm dinh dưỡng TPHCM ở điểm cắt 2,0 chiếm 17,0\% (KTC 95\% 13,3-21,8).

\subsection{Kết cục thai kỳ}

Bảng 3: Đặc điểm kết cuc thai kỳ

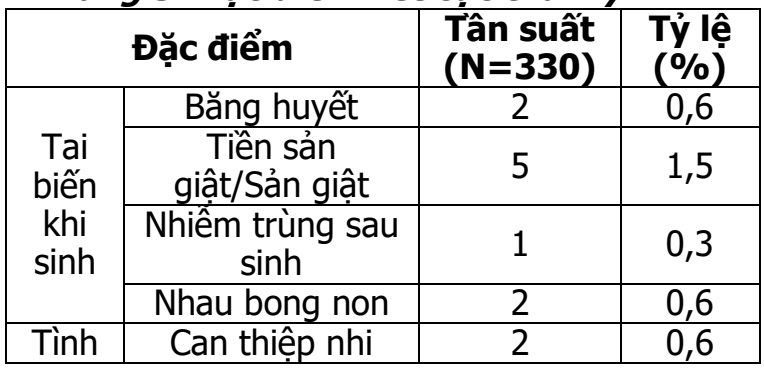

3.4. Mối liên quan giữa dinh dưỡng của thai phụ thừa cân trong thai kỳ và kêt cục thai kỳ của me

Bảng 4: Mối liên quan giữa dinh dướng và kêt cục thai kỳ

\begin{tabular}{|c|c|c|c|c|c|c|}
\hline \multirow{2}{*}{ Kết cục thai kỳ } & \multicolumn{2}{|c|}{ Nguy co } & \multicolumn{2}{|c|}{ Bình thường } & \multirow{2}{*}{$\begin{array}{c}\text { OR } \\
\text { KTC95\% }\end{array}$} & \multirow[b]{2}{*}{$\mathbf{p}$} \\
\hline & $N=56$ & $\%$ & $N=274$ & $\%$ & & \\
\hline \multicolumn{7}{|c|}{ Kết cục thai kỳ của mẹ } \\
\hline Kết cục tốt & 51 & 15,8 & 272 & 84,2 & 1 & \\
\hline Kết cục xấu & 5 & 71,4 & 2 & 28,6 & $13,3(2,5-70,6)$ & 0,000 \\
\hline \multicolumn{7}{|c|}{ Kết cục con } \\
\hline Kết cục tốt & 52 & 16,1 & 270 & 83,9 & 1 & \\
\hline Kết cục xấu & 4 & 50,0 & 4 & 50,0 & $5,2(1,3-21,4)$ & 0,012 \\
\hline
\end{tabular}

Nhận xét: Thai phụ có nguy cơ về dinh dưỡng trong thai kỳ tăng nguy cơ gặp kết cục xâu ở mẹ và bé gấp 5,2 - 13,3 lần so với đối tượng có dinh dưỡng bình thường. Sự khác biệt có ý nghĩa thống kê $(p<0,05)$.

Bảng 5: Mô hình hồi quy đa biên Logistic

\begin{tabular}{|c|c|c|c|}
\hline $\begin{array}{c}\text { Đặc } \\
\text { điểm }\end{array}$ & $\begin{array}{c}\text { OR hiệu } \\
\text { chinh }\end{array}$ & KTC 95\% & p* \\
\hline Dân tộc & & - & \\
\hline Kinh & 1 & - & \\
\hline $\begin{array}{c}\text { Dân tộc } \\
\text { thiếu số }\end{array}$ & 4,8 & $1,01-22,5$ & $\mathbf{0 , 0 4 9}$ \\
\hline
\end{tabular}

\begin{tabular}{|c|c|c|c|}
\hline \multicolumn{4}{|c|}{ Suy dinh dưỡng trước sinh } \\
\hline Không & 1 & - & \\
\hline Có & 64,1 & $11,2-368,3$ & $\mathbf{0 , 0 0 0}$ \\
\hline
\end{tabular}

Nhân xét: Người dân tộc thiếu số có nguy cơ rối loạn dinh dưỡng trong thai kỳ cao gấp 4,8 lần $(\mathrm{OR}=4,8$; KTC 95\%: 1,01 - 22,5) so với thai phụ dân tộc Kinh. Suy dinh dưỡng trước sinh tăng nguy cơ về rối loạn dinh dưỡng cao gấp 64,1 lần (OR=64,1; KTC 95\%: 11,2 - 368,3) so với thai phụ có thể tạng bình thường.

\section{BÀN LUÂ̂N}

Chúng tôi khảo sát 330 đối tượng là sản phụ đến sinh tại bệnh viện đa khoa khu vực Phía Nam Bình Thuận. Tất cả sản phụ này được đánh giá nguy cơ dinh dưỡng bằng thang đo dinh dưỡng của Trung tâm dinh dưỡng TPHCM. Thang đo dinh dưỡng được xây dựng theo các tiêu chuẩn dành riêng cho người Việt Nam dựa vào sự hướng dẫn của Tổ chức Unicef . Kết quả khảo sát cho thấy đối tượng có nguy cơ dinh dưỡng chiếm 17,0\% (KTC 95\% 13,3-21,8) và $83 \%$ (KTC 95\% 78,2 - 86,7) đối tượng có dinh dưỡng bình thường. Điểm trung bình ở nhóm nguy cơ dinh dưỡng và không có nguy cơ dinh dưỡng lần lượt là: $2,04 \pm 0,19$ và $0,36 \pm 0,48$. Chúng tôi nhận thấy tỷ lệ thai phụ có các vấn đề về dinh dưỡng tại bệnh viện đa khoa khu vực phía Nam Bình Thuận còn tương đối cao và đa số các thai phụ có ninguy cơ rối loạn dinh dưỡng khi mang thai thường mắc bệnh thiếu máu hoặc thể trang gầy từ trước khi mang thai. huyết sau sinh, nhau bong non) chiếm tỷ lệ 2,1\%. Qua phân tích đơn biến, chúng tôi tìm thấy mối liên quan giữa các bất thường trong thai kỳ và kết cục thai kỳ không tốt của me đối tượng có nguy cơ về dinh dưỡng trong thai kỳ tăng nguy cơ gặp kết cục xấu ở mẹ gấp 13,3 lần so với đối tượng có dinh dưỡng bình thường (OR=13,3, KTC 95\% 2,5 - 70,6). Do vậy, việc cải thiện tình trạng dinh dương trước sinh và trong khi mang thai rất quan trọng trong việc hạn chế các biến chứng xảy ra cho mẹ và bé
$100 \%$ thai phụ có tình trạng ổn định xuất viện. Thai phụ có kết cục thai kỳ không tốt (băng 
trong quá trình sinh và sau sinh.

Bé sau sinh có cân nặng trung bình là 3054 gram. Bé nhẹ cân nhất là 1900 gram (trường hợp sinh non tháng với tuổi thai 35 tuần ở bà me có bất thường về dinh dưỡng). Chỉ số Apgar bất thường ở 1 phút đầu sau sinh chỉ 4 trường hợp chiếm $(1,2 \%)$. Sau 5 phút hầu hết trẻ có tình trạng ổn định Apgar>7 điểm. Tỷ lệ trẻ nhẹ cân trong nghiên cứu có tỷ lệ tương đối thấp, tuy nhiên bà mẹ có nguy cơ dinh dưỡng trong thai kỳ tăng nguy cơ trẻ nhẹ cân gấp 4,4 lần (KTC95\%: 1,3 -14,9), sự khác biệt có ý nghĩa thống kê $(p<0,05)$. Ghi nhận của chúng tôi gần giống với kết quả của Văn Quang Tân và Lê Thị Hợp thực hiện năm 2012 các thai phụ bị thiếu năng lượng trường diễn có nguy cơ sinh trẻ nhe cân (gấp 3,39 lần, $\mathrm{p}<0,0001$ ) và sinh trẻ có chiều dài dưới $50 \mathrm{~cm}$ (gấp 1,8 lần, $p<0,05$ ) so với các thai phụ không bị thiếu năng lượng trường diễn. Các thai phu bi thiếu máu, có chiều cao dưới $145 \mathrm{~cm}$, nặng dưới $45 \mathrm{~kg}$ trước khi có thai, tăng cân <9kg trong thai kỳ, cân nặng dưới $45 \mathrm{~kg}$ trước khi sinh có nguy cơ sinh trẻ sơ sinh nhẹ cân và chiều dài sơ sinh ngắn cao hơn $(p<0,05)(6)$.

Chúng tôi cũng xác định được mối liên quan giữa dinh dưỡng trong thai kỳ của me và kêt cục thai kỳ của trẻ $(p<0,05)$. Bà me có nguy cơ dinh dưỡng trong thai kỳ tăng nguy cơ con gặp kết cục thai kỳ xấu (vàng da nặng sau sinh) gấp 5,2 lần (KTC95\% 1,3-21,4).

Khi tiến hành phân tích hồi quy đa biến, chúng tôi ghi nhận 2 yếu tố làm tăng nguy cơ bất thường ở thai kỳ bao gồm, đối tượng là người dân tộc thiểu số có nguy cơ gặp bất thường về dinh dưỡng trong thai kỳ cao gấp 4,8 lần $(\mathrm{OR}=4,8$; $\mathrm{KTC} 95 \%$ : 1,01-22,5) so với các thai phụ thuộc dân tộc Kinh. Phụ nữ có suy dinh dưỡng trước sinh tăng nguy cơ có rối loạn về dinh dưỡng cao gấp 64,1 lần (OR=64,1; KTC 95\%: 11,2-368,3) phụ nữ có thể tạng bình thường. Kết quả của chúng tôi cũng tương tự với kết quả trong nghiên cứu của Canaan Negash khảo sát tại vùng nông thôn tại Hula, Ethiopia cho thấy BMI của bà me có liên quan đến tình trạng dinh dưỡng của trẻ (7). Với cũng kết luận, tác giả Kumar Guru Mishra thực hiện nghiên cứu tại Odisha, Ấn Độ thông qua khảo sát 418 phụ nữ mang thai được theo dõi cho đến khi sinh, và đánh giá cân nặng của mẹ, tăng cân trong suốt thai kỳ và thai nhi. Tác giả Mishra cũng ghi nhân có sự liên quan về tình trạng suy dinh dương ở bé khi phụ nữ mang thai có BMI thấp $(p=0,03)$
(8). Chúng tôi nhận thấy trong nghiên cứu của mình, đối tượng nguy cơ gặp bất thường dinh dưỡng trong thai kỳ là đối tượng phụ nữ người dân tộc thiểu số có nhẹ cân, hoặc suy dinh dưỡng trước sinh. Vì vậy, việc nâng cao tuyên truyền sức khỏe rộng rãi trong đồng bào dân tộc sống ở khu vực vùng sâu, vùng xa đặt ra nhiều thách thức cho ngành $Y$ tế dự phòng tại địa phương.

\section{KẾT LUÂ̂N}

Việc áp dung thang đo dinh dưỡng của trung tâm dinh dưỡng TPHCM là khả thi trong sàng lọc các thai phụ. Thông qua điểm cắt $2,0 \mathrm{khi}$ sử dung thang đo dinh dưỡng, chúng tôi phân lập được nhóm thai phụ có nguy cơ rối loạn dinh dưỡng tại thời điểm sinh và xử lý kịp thời các ảnh hưởing xấu xảy ra cho bà me và bé sơ sinh. Nên áp dụng thang đo dinh dưỡng nhằm đánh giá tình trạng dinh dưỡng cho tất cả thai phụ trong thai kỳ và sau sinh tại các bệnh viện, đặc biệt ở các bệnh viện tuyến cơ sở.

\section{TÀI LIẸU THAM KHẢO}

1. Bộ Y tế (2017), Hướng dẫn quốc gia về dinh dưỡng cho Phụ nữ có thai và bà mẹ cho con bú Ban hành kèm theo Quyết định số 776/QĐ-BYT ngày 08 tháng 3 năm 2017 của Bộ trưởng Bộ Y tế, Vụ Sức khỏe Bà me - Trẻ em Hà Nội.

2. Lanzone A Triunfo S (2015), "Impact of maternal under nutrition on obstetric outcomes", ] Endocrinol Invest. 38(1), pp $31-8$.

3. Lê Thị Thanh Hoa, Lế Thị Hương Nguyễn Thị Thanh Tâm (2014), "Tình trạng dinh dướng và một số yếu tố liên quan của phụ nữ mang thai huyện Duy Tiên, tỉnh Hà Nam năm 2014", Tạp chí DD\&TP/Journal of Food and Nutrition Sciences.

4. Bộ Y tế (2015), Hướng dẫn chẩn đoán và điều trị các bệnh sản phụ khoa - Ban hành kèm theo Quyết định số 315/QĐ-BYT ngày 29/01/2015, Hà Nội, Cục Quản lý Khám chữa bệnh.

5. Trung tâm dinh dưỡng TPHCM (2019), Mẫu 02 - TTDD Phiếu dánh giá tình trạng dinh dưỡng do Trung tâm Dinh dưỡng TPHCM xây dựng dưới sự hỗ trợ của UNICEF tại Việt Nam, TPHCM, Trung tẩm Dinh dưỡng TPHCM.

6. Văn Quang Tân và Lể Thi Hợp (2012), "Thực trạng dinh dướng của bà mẹ và chiêu dài cấn nặng của trẻ sơ sinh tại tỉnh Bình Dương năm 2012", Tạp chí DD\&TP/journal of Food and Nutrition Sciences.

7. C. Negash (2015),"Association between Maternal and Child Nutritional Status in Hula, Rural Southern Ethiopia: A Cross Sectional Study", PLoS One. 10(11), e0142301.

8. K. G. Mishra, V. Bhatia R. Nayak (2020), "Maternal Nutrition and Inadequate Gestational Weight Gain in Relation to Birth Weight: Results from a Prospective Cohort Study in India", Clin Nutr Res. 9(3), 213-222. 